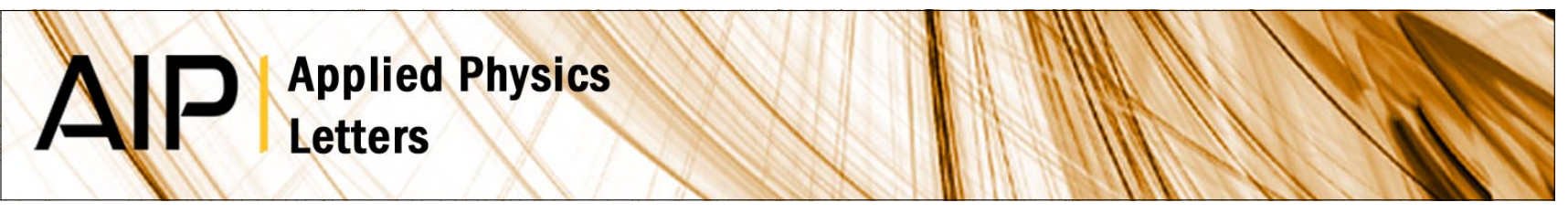

\title{
Tunneling of holes is observed by second-harmonic generation
}

M. K. Vanbel, V. V. Afanas'ev, C. Adelmann, M. Caymax, V. K. Valev et al.

Citation: Appl. Phys. Lett. 102, 082104 (2013); doi: 10.1063/1.4793578

View online: http://dx.doi.org/10.1063/1.4793578

View Table of Contents: http://apl.aip.org/resource/1/APPLAB/v102/i8

Published by the American Institute of Physics.

\section{Related Articles}

Bandstructure measurement in nonlinear optical waveguide arrays

Appl. Phys. Lett. 102, 081104 (2013)

Note: Auto-relock system for a bow-tie cavity for second harmonic generation

Rev. Sci. Instrum. 84, 026111 (2013)

Application of smooth exterior scaling method to calculate the high harmonic generation spectra J. Chem. Phys. 138, 074313 (2013)

Computational analysis of the quadrupole contribution in the second-harmonic generation spectroscopy for the water/vapor interface

J. Chem. Phys. 138, 064704 (2013)

Harnessing second-order optical nonlinearities at interfaces in multilayer silicon-oxy-nitride waveguides

Appl. Phys. Lett. 102, 061106 (2013)

\section{Additional information on Appl. Phys. Lett.}

Journal Homepage: http://apl.aip.org/

Journal Information: http://apl.aip.org/about/about_the_journal

Top downloads: http://apl.aip.org/features/most_downloaded

Information for Authors: http://apl.aip.org/authors

\section{ADVERTISEMENT} \section{AIP $\begin{aligned} & \text { Applied Physics } \\ & \text { Letters }\end{aligned}$}

\section{EXPLORE WHAT'S NEW IN APL}

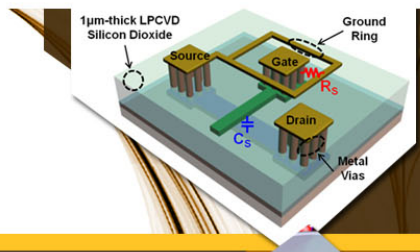

SURFACES AND INTERFACES

Focusing on physical, chemical, biological structural, optical, magnetic and electrical properties of surfaces and interfaces, and more.. 


\title{
Tunneling of holes is observed by second-harmonic generation
}

\author{
M. K. Vanbel, ${ }^{1}$ V. V. Afanas'ev, ${ }^{2}$ C. Adelmann, ${ }^{3}$ M. Caymax,${ }^{3}$ V. K. Valev,${ }^{4}$ and T. Verbiest ${ }^{1}$ \\ ${ }_{1}^{1}$ Molecular and Nanomaterials, Department of Chemistry, KU Leuven, 3001 Leuven, Belgium \\ ${ }^{2}$ Semiconductor Physics, Department of Physics, KU Leuven, 3001 Leuven, Belgium \\ ${ }^{3}$ Imec, Kapeldreef 75, 3001 Leuven, Belgium \\ ${ }^{4}$ Cavendish Laboratory, Department of Physics, University of Cambridge, J. J. Thomson Avenue, \\ Cambridge CB3 OHE, United Kingdom
}

(Received 17 December 2012; accepted 13 February 2013; published online 27 February 2013)

\begin{abstract}
$\mathrm{Al}_{2} \mathrm{O}_{3}$ passivated $\mathrm{n}$-doped $\mathrm{Si}$ substrates are investigated by second-harmonic generation (SHG) upon applying an external electric field by a corona wire. The observed change in the SHG response upon applying an external electric field is attributed to charge transfer in the semiconductor. Capacitance-voltage measurements are performed to affirm this conclusion. Upon applying a large negative electric field over the structure, a clear alteration in SHG signal is observed, which corresponds to tunneling of holes from the n-doped silicon into the aluminum oxide layer. ㅇ 2013 American Institute of Physics. [http://dx.doi.org/10.1063/1.4793578]
\end{abstract}

The optimization of the channel in a metal oxide semiconductor (MOS) is one of the key issues in the semiconductor industry. The function of an MOS device is to alter the current through the channel (or space-charge region in the semiconductor), which results in an on-current or offcurrent. In an actual device, the switching between on and off is achieved by changing the voltage on the metal gate. Besides electrical techniques, this switching between on and off can be investigated by second-harmonic generation (SHG) and electric field-induced SHG (EFISH), with the advantage that it does not necessarily require the fabrication of electrodes on the structure. Moreover, SHG gives information on both the electrical properties and interface properties. Investigation of the space-charge region (SCR) of silicon and the interface properties of semiconductors with metal gate by means of EFISH has been evaluated by many authors, such as Aktsipetrov et al., ${ }^{1,2}$ Chang et al., ${ }^{3}$ Glinka et al., ${ }^{4}$ and Scheidt et al. ${ }^{5,6}$ Moreover, SHG has been used to characterize the interface of MOS structures. ${ }^{7,8}$ Although MOS structures form the basis of the current transistor devices, quantum well devices are promising to keep scaling the devices according to the scaling laws. ${ }^{9,10}$ In these devices, the tunneling current is of critical importance for device operation. Since the tunneled charges are confined in the structure, the SHG response can give valuable insights into quantum well devices.

However, in the previously mentioned investigations, ${ }^{1,3}$ the SHG contributions from the metal layer could not be separated from those of the charges at the interface.

In order to clearly investigate the role of the SHG signal from the relevant semiconductor interface, we chose a different approach to charge the structure, namely by positioning a charged corona wire above the aluminum oxide passivated semiconductor. In this manner, no additional metal layer is present, which in turn cannot contribute to the total (SHG) signal and facilitates the analysis.

In this letter, we report the SHG response upon changing the electric corona field from zero to positive or negative and vice versa. Applying a positive corona field decreases the initial electric field in the space-charge region of $\mathrm{Si}$, decreasing the SHG signal. Upon applying a large negative electric field over the structure, a clear increase and subsequent decrease in SHG signal is observed, where the latter corresponds to tunneling of holes through the $\mathrm{SiO}_{\mathrm{x}}$ layer. The secondharmonic signals are verified by capacitance-voltage measurements on the same structures, to justify the physical model that is proposed here. The change in SHG intensity is correlated to the exponential tunneling decay and the decrease of electric field in the silicon SCR due to dissipation of the corona charges at the surface.

The $\mathrm{Si}-\mathrm{SiO}_{2}-\mathrm{Al}_{2} \mathrm{O}_{3}$ structures were prepared on $300 \mathrm{~mm}$ n-type Si (100) wafers. Initially, a $\sim 200 \mathrm{~nm}$ thick As-doped epitaxial Si layer was deposited by chemical vapor deposition in an ASM Epsilon reactor. The doping level was $10^{16} \mathrm{~cm}^{-3}$. Subsequently, a $\sim 1 \mathrm{~nm}$ thick chemical $\mathrm{SiO}_{2}$ was grown on the surface in ozonated $\mathrm{H}_{2} \mathrm{O}$. Different thickness of $\mathrm{Al}_{2} \mathrm{O}_{3}$ was then deposited by atomic layer deposition in an ASM Pulsar reactor from trimethyl aluminum and $\mathrm{H}_{2} \mathrm{O}$ at $300{ }^{\circ} \mathrm{C}$.

The time-dependent second-harmonic generation (TD-SHG) experiments were carried out using a Ti- $\mathrm{Al}_{2} \mathrm{O}_{3}$ laser at the fixed fundamental wavelength of $800 \mathrm{~nm}$ with an average power output of $70 \mathrm{~mW}$. The pulse length and repetition rate are, respectively, $120 \mathrm{fs}$ and $82 \mathrm{MHz}$. For our measurements, the polarizer-analyzer combination of $\mathrm{p}_{\text {in }}-\mathrm{p}_{\text {out }}$ was used. ${ }^{11}$

The charging of the passivated semiconducting samples is achieved by corona poling, as is schematically presented in Fig. 1. The corona wire was placed at $3 \mathrm{~cm}$ from the sample. A voltage of $-3 \mathrm{kV}$ and $2.7 \mathrm{kV}$ was used to charge the surface, which did not lead to oxide breakthrough. Upon charging, the voltage was quickly ramped from $0 \mathrm{~V}$ to the maximal value. The current from the corona wire to the substrate was monitored and remained constant during the measurements.

In centrosymmetric materials like $\mathrm{Si}, \mathrm{SHG}$ is forbidden within the electric dipole approximation. However, the total SHG response of uncharged structures can be written in terms of electric dipole contributions $\vec{P}^{D}(2 \omega)$ and electric quadrupole contributions $\vec{P}^{Q}(2 \omega)$ by 


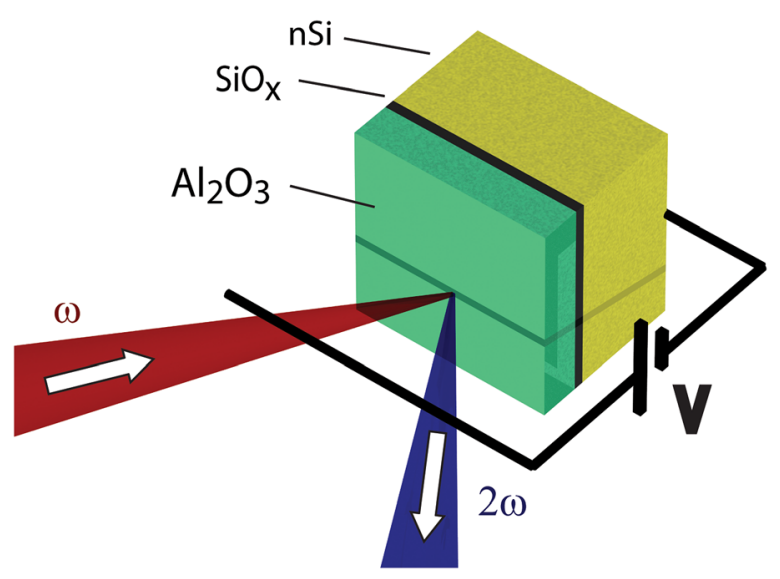

FIG. 1. Schematic representation of the TD-SHG experiment on aluminum oxide passivated silicon with a corona wire. An external electric field can be applied between the surface of the metal-oxide-semiconductor structure and the corona wire. Due to this electric field, an internal field in the spacecharge region in silicon is induced.

$$
\begin{aligned}
\vec{P}^{e f f}(2 \omega) & =\vec{P}^{D}(2 \omega)+\vec{P}^{Q}(2 \omega) \\
& =\chi^{D}: \vec{E}(\omega) \vec{E}(\omega)+\chi^{Q}: \vec{E}(\omega) \nabla \vec{E}(\omega),
\end{aligned}
$$

where $\chi^{D}$ and $\chi^{Q}$ indicate the dipolar and the quadrupole nonlinear susceptibilities. A separation of the dipolar and quadrupole contributions is usually not possible in an experiment with a single fundamental beam in a polarizer-analyzer combination of $p_{\text {in }}-p_{\text {out }}$. Hence, the nonlinear polarization attributed to $\mathrm{Si}$ will be treated as an effective polarization.

Furthermore, upon applying an EFISH term can be written as

$$
\vec{P}^{d c-\text { induced }}(2 \omega)=\chi^{(3)}: \vec{E}(\omega) \vec{E}(\omega) \vec{E}_{d c},
$$

where $\vec{E}_{d c}$ is the internal electric field, arising from the external applied electric field and $\chi^{(3)}$ is a fourth rank tensor. Since $\vec{E}_{d c}$ is oriented in the $\mathrm{z}$ direction only, Eq. (2) can be written as

$$
\vec{P}_{e f f}^{d c-\text { induced }}(2 \omega)=\chi_{e f f}^{(3)}: \vec{E}(\omega) \vec{E}(\omega) \vec{E}_{z, d c}
$$

where $\vec{E}_{z, d c}$ is only oriented in the z-direction, perpendicular to the surface and $\vec{P}_{e f f}$ denotes that we neglect retardation effects. ${ }^{1}$

From Eqs. (1) and (3), the total SHG polarization and SHG intensity in reflection can be described as ${ }^{1,12,13}$

$$
\begin{aligned}
\vec{P}_{\text {total }}= & \chi^{D}: \vec{E}(\omega) \vec{E}(\omega)+\chi^{Q}: \vec{E}(\omega) \nabla \vec{E}(\omega) \\
& +\chi^{(3)}: \vec{E}(\omega) \vec{E}(\omega) \vec{E}_{z, d c} \\
I_{(2 \omega)} \sim & \left(\vec{P}_{\text {total }}\right)^{2}
\end{aligned}
$$

where $\chi^{D}$ reflects the centrosymmetry of the structure and the symmetry of surface or interface, $\chi^{Q}$ reflects the crystal lattice and the gradient of the light within the penetration depth, and $\chi^{(3)}$ corresponds to EFISH. Only $\chi^{(3)}$ is affected by the applied electric field, while $\chi^{D}$ and $\chi^{Q}$ stay invariant. The major contribution in $\chi^{(3)}$ arises mostly from the $\chi_{z z z z}$ and $\chi_{z x x z}=\chi_{z y y z}$, since the direction of the electric field in the SCR of silicon is in the z-direction.
Upon applying a corona field, only the $\vec{E}_{d c}$ term will be affected. The number of charges particles $Q(t)$ can be written as

$$
Q(t)=q \cdot N_{t}\left(1-\exp \left(\frac{-t \cdot I_{e} \cdot \sigma}{q}\right)\right)
$$

where $q$ is the charge, $N_{t}$ is the number of charges, $\mathrm{t}$ is the time, $I_{e}$ is the current of the charges in the SCR of the semiconductor, and $\sigma$ is the capture cross section. The electric field in the SCR $\vec{E}(t)$ in a semiconductor can be written as

$$
\vec{E}(t) \sim \vec{E}(0)+A \cdot\left(1-\exp \left(\frac{-t \cdot I_{e} \cdot \sigma}{q}\right)\right),
$$

where $A$ describes the amplitude of decay, which is dependent on structure design.

In equilibrium, $I_{e}$ is equal to the current of ions at the surface due to the corona charging. Hence, for a decrease in electric field in the SCR due to tunneling, the EFISH intensity according to Eq. (4) can be written as

$$
\begin{aligned}
I_{E F I S H(2 \omega)} & \sim\left(\vec{P}_{E F I S H}\right)^{2} \\
& \sim\left(\vec{E}(0)+A \cdot\left(1-\exp \left(\frac{-t \cdot I_{e} \cdot \sigma}{q}\right)\right)\right)^{2} .
\end{aligned}
$$

When the corona field is turned off, due to dissipation of the corona charges, the electric field in the SCR of the semiconductor obeys ${ }^{14}$

$$
\vec{E}(t)=\beta \cdot \ln \left(B\left(t+t_{0}\right)\right)^{-1},
$$

where $\beta$ is the field dependency with SCR thickness, $B=C \beta / \varepsilon_{i}, \mathrm{C}$ is the capacitance, $\varepsilon_{i}$ is the insulator dielectric constant, and $t_{0}$ is the constant of integration obtained from $\vec{E}(0)$.

From Eq. (4), the EFISH intensity upon turning the corona field off can be expressed as

$$
I_{\operatorname{EFISH}(2 \omega)} \sim\left(\vec{P}_{E F I S H}\right)^{2} \sim\left(\beta \cdot \ln \left(B\left(t+t_{0}\right)\right)^{-1}\right)^{2} .
$$

It should be mentioned that the oxide thickness influences the electric field, since a voltage drop appears over the oxide layer; however, this decrease in field is small compared to the applied corona field. Moreover, fixed charges in $\mathrm{Al}_{2} \mathrm{O}_{3}$ and interface charges influence the dc-induced polarization. Furthermore, multiple reflections in the oxide layer are neglected, since the SHG intensity is similar for all the samples with different oxide thickness and is predicted to be small over the range of $20 \mathrm{~nm}$, under non-Brewster angle conditions. $^{15}$

The SHG intensity of the $\mathrm{Si}-\mathrm{SiO}_{\mathrm{x}}-\mathrm{Al}_{2} \mathrm{O}_{3}$ structures is shown in Fig. 2. Initially, the SHG intensity corresponds to an uncharged situation. In situation (a) in Fig. 2, the uncharged sample is schematically represented, where the direction and the strength of the electric field in the SCR are represented with an arrow. Upon applying a positive external field at $\mathrm{t}=0 \mathrm{~s}$, the SHG response decreases dramatically, which indicates that the electric field in the Si SCR decreased as well, according to Eq. (4). Due to the corona 

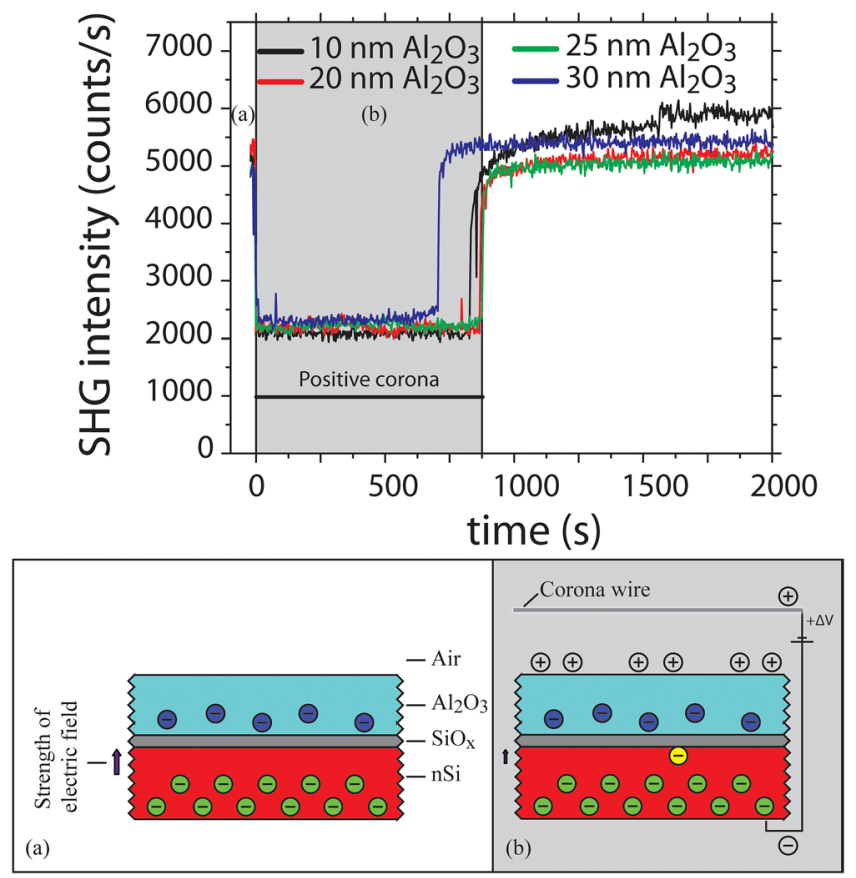

FIG. 2. SHG response upon applying a positive corona field to an $\mathrm{Al}_{2} \mathrm{O}_{3}$ passivated Si wafer. The initial SHG response corresponds to the uncharged sample. This situation is schematically represented in situation (a). The strength of the electric field in the SCR of Si is represented by the magnitude of the arrow. At $0 \mathrm{~s}$, a positive corona field of $2.7 \mathrm{kV}$ is applied, resulting in a drop in SHG intensity. The negative doping charges are attracted to the interface, shown in situation (b). After several hundreds of seconds, the corona field was turned off, resulting in a recovery of the SHG signal to its original intensity.

field, electrons from the silicon bulk are attracted to the $\mathrm{Si}$ / oxide interface, resulting in a decrease in field in the silicon space-charge region (situation (b) in Fig. 2). Upon increasing the corona field, no drastic change is observed in the structure, only an increase of the amount of charges near the interface. At $700 \mathrm{~s}$ or $860 \mathrm{~s}$, depending on the sample, the electric field is switched off, whereby the SHG signal recovers to its original intensity, i.e., the intensity level without electric field. Hence, the charge distribution recovers to the original structure and the field in the SCR increases due to the dissipation of charges. It can be clearly observed that the charging effect is equal for all structures, independent of oxide thickness. This is easily explained, because the voltage drop over the oxide layer is small, compared to the corona field that is used to charge the surface. The initial (before corona charging) and final (after removal of the corona field) SHG intensities are equal, indicating that the electrical properties in the Si-SCR have not been altered by the measurements, which is confirmed by capacitance-voltage (CV) measurements shown in Fig. 4. For the positive corona, the hysteresis difference before and after the charging is similar. Moreover, the positive flat-band potential is not shifted. From this, we can conclude that the initial and final amounts of charges in the oxide layer are equal and that the small amount of charges present in the oxide layer is negative.

Applying a negative corona field induces a drastic increase in SHG intensity compared to the uncharged initial intensity (Fig. 3). Due to the negative corona field, electrons are removed from the SCR, and minority carriers (i.e., holes) are attracted to the interface. The minority carriers increase

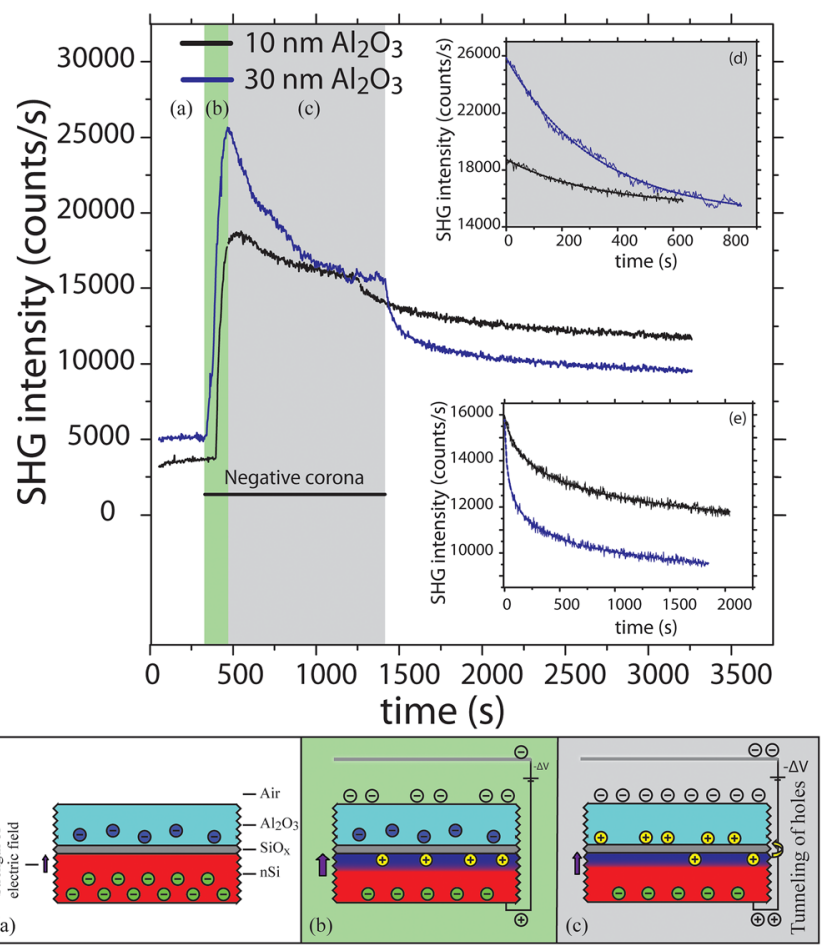

FIG. 3. SHG response upon applying a negative corona field to an $\mathrm{Al}_{2} \mathrm{O}_{3}$ passivated $\mathrm{Si}$ wafer. Similar curves were obtained for another thickness of the $\mathrm{Al}_{2} \mathrm{O}_{3}$ layer. The initial SHG response corresponds to the uncharged sample. This situation is schematically represented in situation (a). The strength of the electric field in the SCR of $\mathrm{Si}$ is represented by the magnitude of the arrow. Upon applying a $-3 \mathrm{kV}$ corona field, a large increase in SHG intensity is observed. This correlates to the increase in electric field in the space-charge region of $\mathrm{Si}$, shown in situation (b). From a certain electric field over the oxide layer, tunneling of holes from $\mathrm{Si}$ to $\mathrm{Al}_{2} \mathrm{O}_{3}$ occurs, which reduces the effective electric field in the silicon space-charge region. Hence, the SHG intensity drops exponentially, as predicted by theory. Schematically, the situation is described by situation (c). Upon switching the corona charge off, the SHG drops logarithmically due to the removal of charges in the space-charge region in Si. However, the SHG signal does not recover to the initial intensity due to remaining positive charges in the oxide layer, enabling a larger electric field in the silicon spacecharge region. In inset (d), the fitting on the time-shifted tunneling to an exponential decay of situation (c) is shown. Similar relaxation times (313 s vs. 320 s) are obtained for both the $10 \mathrm{~nm}$ and $30 \mathrm{~nm}$ thick oxide layer. In inset (e), the logarithmic decay upon turning the corona field off is shown.

dramatically the electric field, inducing a large electric field built-up in the Si space-charge region. Due to the positive nature of the charges, a larger field arises in the silicon SCR. This results in an increase in SHG intensity, which is described in situation (b) in Fig. 3. However, after a few tens of seconds, the SHG intensity starts to decrease exponentially. The observed decrease in SHG intensity can be explained by tunneling, since the electric field over the $\mathrm{SiO}_{\mathrm{x}}$ layer becomes too large. The internal electric field in the SCR of silicon decreases exponentially, according to Eq. (6). Positive charges tunnel through the $\mathrm{SiO}_{\mathrm{x}}$ layer, resulting in a decrease of minority carriers in the Si SCR. According to Eq. (7), the EFISH contribution of this electric field decreases as well (shown in inset (d) in Fig. 3). Schematically, the situation is represented in situation (c) in Fig. 3. Moreover, when corona field is turned off and the charges dissipate, the change in SHG intensity can be described by Eq. (9). The fittings are shown in inset (e) in Fig. 3. Furthermore, the final SHG response is considerably larger than the initial value, suggesting that the structure is 


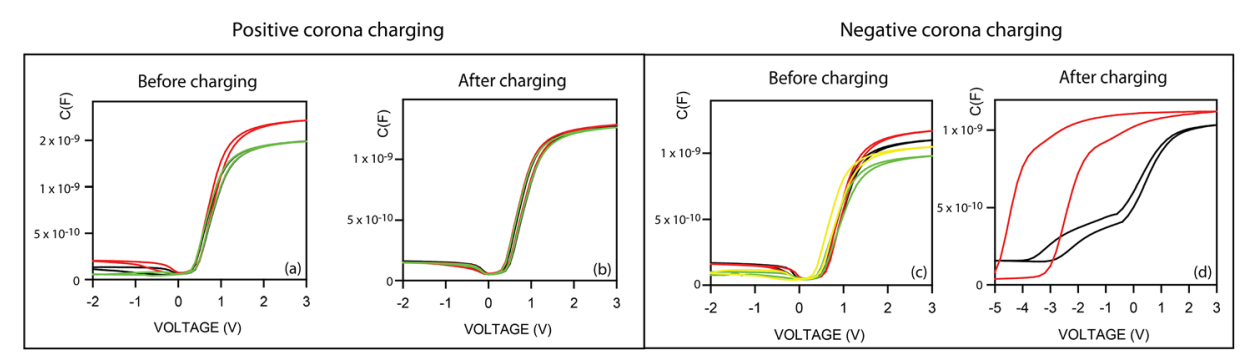

FIG. 4. Capacitance-voltage characteristics before and after negative corona poling. For the positive corona charging, there is no significant difference in the $\mathrm{CV}$ response ((a) vs (b)), confirming that the structure was not electrically altered during the corona poling. For the negative corona, there is a clear difference in CV response ((c) vs (d)), indicating that there is an electrical difference between the samples before and after poling. Due to the shift in flat-band potential and the increase in hysteresis in (b), it is clear that the amount of charges in the oxide layer increases and change sign to positive charges.

electrically changed during the measurement. The effect of tunneling can be confirmed by $\mathrm{CV}$ measurements, shown in Fig. 4. The CV characteristics of the charged structure exhibit a shift to negative voltage and a large hysteresis. Due to this shift of flat-band potential and large hysteresis, the positive nature of the charges in the oxide can be confirmed. Hence, holes tunneled from the $\mathrm{Si}$ layer to the $\mathrm{Al}_{2} \mathrm{O}_{3}$ layer and remained in the oxide layer. This results in a larger field in the space-charge region, enabling a larger final SHG response, compared to the initial SHG response.

We show that, due to changes in charge density in the space-charge region of silicon, the time-dependent second-harmonic generation response of a structure can be altered. Furthermore, tunneling of holes under the influence of a positive corona field is observed with second-harmonic generation, which is confirmed by capacitance-voltage measurements. Previous methods of electric-field induced secondharmonic generation could not separate the influence of the metal layer and the important Si space-charge region, while our method can. This approach can lead to valuable insights into quantum well devices.

This work was supported by the Katholieke Universiteit Leuven (GOA). M.K.V. acknowledges the useful scientific discussions with W. Brullot, S. Vandendriessche, and M. Bloemen. V.K.V. is grateful for the financial support from the fund for scientific research Flanders (FWO-Vlaanderen).
${ }^{1}$ O. A. Aktsipetrov, A. A. Fedyanin, E. D. Mishina, A. N. Rubtsov, C. W. van Hasselt, M. A. C. Devillers, and Th. Rasing, Phys. Rev. B 54, 1825 (1996).

${ }^{2}$ O. A. Aktsipetrov, A. A. Fedyanin, A. V. Melnikov, J. I. Dadap, X. Y. Hu, M. H. Anderson, M. C. Downer, and J. K. Lowell, Thin Solid Films 294, 231 (1997).

${ }^{3}$ C.-L. Chang, W. C. Lee, L. K. Chu, M. Hong, J. Kwo, and Y.-M. Chang, Appl. Phys. Lett. 98, 171902 (2011).

${ }^{4}$ Y. D. Glinka, W. Wang, S. K. Singh, Z. Marka, S. N. Rashkeev, Y. Shirokaya, R. Albridge, S. T. Pantelides, and N. H. Tolk, Phys. Rev. B 65, 193103 (2002).

${ }^{5}$ T. Scheidt, E. G. Rohwer, P. Neethling, H. M. von Bergmann, and H. Stafast, J. Appl. Phys. 104, 083712 (2008).

${ }^{6}$ T. Scheidt, E. G. Rohwer, and H. W. von Bergmann, Phys. Rev. B 69, 165314 (2004).

${ }^{7}$ V. K. Valev, M. K. Vanbel, B. Vincent, V. V. Moshchalkov, M. Caymax, and T. Verbiest, IEEE Electron Device Lett. 32, 12 (2011).

${ }^{8}$ M. K. Vanbel, V. K. Valev, B. Vincent, V. V. Afanas'ev, J.-P. Locquet, S. Van Elshocht, M. Caymax, and T. Verbiest, J. Appl. Phys. 111, 064504 (2012).

${ }^{9}$ L. Gomez, P. Hashemi, and J. L. Hoyt, IEEE Trans. Electron Devices 56, 2644 (2009).

${ }^{10}$ W. Yu, B. Zhang, Q. T. Zhao, J.-M. Hartmann, D. Buca, A. Nichau, R. Lupták, J. M. Lopes, S. Lenk, M. Luysberg, K. K. Bourdelle, X. Wang, and S. Mantl, Solid State Electron. 62, 185 (2011).

${ }^{11}$ M. K. Vanbel, A. Delabie, S. Sioncke, C. Adelmann, V. V. Afanas'ev, J.-P. Locquet, S. Van Elshocht, M. Caymax, and T. Verbiest, Proc. SPIE 8434, 84341F (2012).

${ }^{12}$ Y. R. Shen, The Principles of Non-linear Optics (Wiley, New York, 1992), pp. 42-52.

${ }^{13}$ T. F. Heinz, H. W. K. Tom, and Y. R. Shen, Phys. Rev. A 28, 1883 (1983).

${ }^{14}$ Z. A. Weinberg, Solid-State Electron. 20, 11 (1977).

${ }^{15}$ C. W. van Hasselt, M. A. C. Devillers, Th. Rasing, and O. A. Aktsipetrov, J. Opt. Soc. Am. B 12, 33 (1995). 